\title{
Taxonomic status of Apostolepis barrioi Lema, 1978, with comments on the taxonomic instability of Apostolepis Cope, 1862 (Serpentes, Dipsadidae)
}

\author{
Omar Machado Entiauspe-Neto', Arthur de Sena², \\ Arthur Tiutenko ${ }^{3}$, Daniel Loebmann'
}

I Universidade Federal do Rio Grande, Instituto de Ciências Biológicas, Laboratório de Vertebrados, Av. Itália Km 8, CEP: 96203-900, Vila Carreiros, Rio Grande, Rio Grande do Sul, Brazil 2 Universidade do Estado de Mato Grosso, Departamento de Biologia. CEP: 78690-000, Nova Xavantina, Mato Grosso, Brazil 3 University of Erlangen-Nuremberg, Schlossplatz 6, D-91054 Erlangen, Germany

Corresponding author: Omar Machado Entiauspe-Neto (omarentiauspe@hotmail.com)

Academic editor: Robert Jadin | Received 27 January 2019 | Accepted 10 March 2019 | Published 23 April 2019

http://zoobank.org/278689B7-CEOC-47EF-A359-FB1E097B442D

Citation: Entiauspe-Neto OM, de Sena A, Tiutenko A, Loebmann D (2019) Taxonomic status of Apostolepis barrioi Lema, 1978, with comments on the taxonomic instability of Apostolepis Cope, 1862 (Serpentes, Dipsadidae). ZooKeys 841: 71-78. https://doi.org/10.3897/zookeys.841.33404

\begin{abstract}
Apostolepis is a diverse neotropical snake genus, which has been historically subjected to poor taxonomic descriptions, largely based on either a small type series or subjective diagnoses. We evaluate the case of Apostolepis barrioi Lema, 1978 and its intricate taxonomic history, suggesting its synonymization with Apostolepis dimidiata (Jan, 1862), and providing brief commentary on the taxonomic instability that has been plaguing the genus.
\end{abstract}

\section{Keywords}

Elapomorphini, Neotropical, synonymy, taxonomy

\section{Introduction}

The Neotropical dipsadid snake genus Apostolepis Cope, 1862 comprises over 30 species, with an even broader synonym list, being marked by a systemic proliferation of "poorly defined" taxa, described based on single or few individuals with poor documentation of

Copyright O. M. Entiauspe-Neto et al. This is an open access article distributed under the terms of the Creative Commons Attribution License (CC BY 4.0), which permits unrestricted use, distribution, and reproduction in any medium, provided the original author and source are credited. 
variation (Vanzolini 1986; Ferrarezzi 1993; Harvey 1999; Ferrarezzi et al. 2005; Nogueira et al. 2012). Apostolepis dimidiata (Jan, 1862) is a small-sized fossorial snake that occurs in the Cerrado, Chaco and Atlantic Forests at Argentina, Brazil and Paraguay (Cei 1993; Giraudo and Scrocchi 1998; Harvey 1999). Jan (1862) described Elapomorphus dimidiatus based on a specimen from "Brazil", and allocated it to the subgenus Elapomojus Jan, 1862. Later, Peters (1880) described Elapomorphus erythronotus based on a specimen from "São Paulo" in southeastern Brazil. Cope (1887) presented two brief taxon descriptions, Apostolepis erythronotus lineatus and Rhynchonyx ambiniger vittatus, both from Chapada dos Guimarães, Mato Grosso, in central-western Brazil. Boulenger (1896) elevated both of Cope's subspecies to species level and placed Elapomorphus erythronotus in Apostolepis. Werner (1897) described Apostolepis nigriceps based on two specimens, of which only one has a known locality given as "São Paulo", in southeastern Brazil. Lema (1978) described three new species for Paraguay: Apostolepis barrioi from the Ypané River, Cororo, Concepcíon Province; Apostolepis ventrimaculatus from "Paraguay"; and Apostolepis villaricae from Villa Rica, Concepcíon Province. Later, Lema (1986) would synonymize $A$. erythronota, $A$. nigriceps and $A$. ventrimaculatus with $A$. dimidiata, while also revalidating $A$. lineata. Lema (1993) presented a review on the morphological variation of $A$. dimidiata, while also allocating the species he previously described, $A$. barrioi and $A$. ventrimaculatus, as synonyms of the former.

\section{Taxonomy}

Recently, Cabral et al. (2017) presented a revalidation of $A$. barrioi, diagnosing it from all congeners based on an immaculate white venter, narrow dorsolateral stripes not in contact with the ventrals, and a terminal black shield. There is also a wide overlap between the meristic variation of $A$. barrioi and $A$. dimidiata, such as in the number of ventral scales (222-256 in A. barrioi; 214-264 in A. dimidiata) and subcaudal scales (23-55 in A. barrioi; 22-39 in A. dimidiata), in its morphometric variation (given in $\mathrm{mm}$ ), in snout-vent length (188-542 in A. barrioi; 180-676 in $A$. dimidiata) and tail length (16-45 in $A$. barrioi; 16-60 in A. dimidiata), and geographic variation, since both species are sympatric along their whole distribution, as reported by the authors (Cabral et al. 2017: 246). Furthermore, the authors present a comparative table of Apostolepis species in which A. barrioi is stated as having an immaculate venter and A.dimidiata, a venter heavily pigmented with black, having only the edge of the ventrals white. This is in clear conflict with the original description, considering that the holotype of $A$. dimidiata presented an immaculate yellow venter according to the original description "[...] parte inferiore del corpo é giallastra, meno la testa che inferiormente ha del nero sugli inframascellari e sulle squame che stanno in vicinanzi ai sottolabiali" (En: lower part of the body is yellowish, except for the head, that has black inferiorly, in the inframaxillary (region) and in the scales near the infralabials) (Jan 1862: 48). Unfortunately, this specimen (holotype of $A$. dimidiata) could not be examined, since it was destroyed during the Second World War. It is also relevant that Lema (1993: 47) 
A

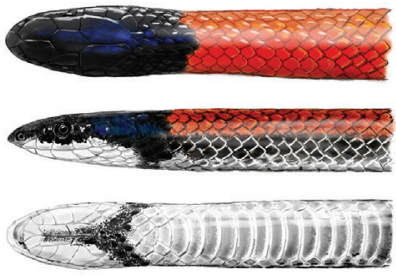

B

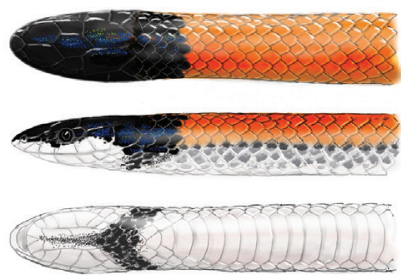

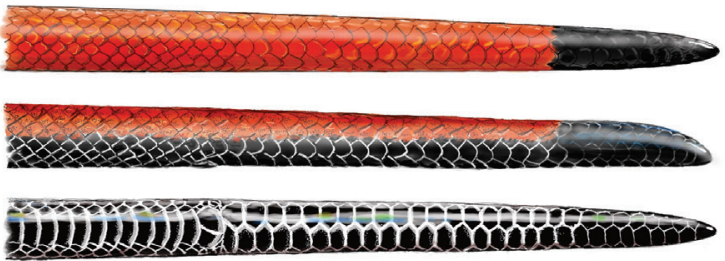

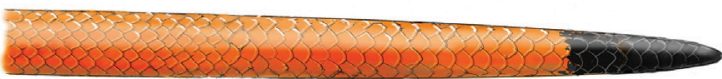

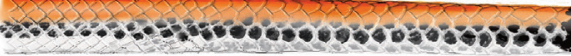

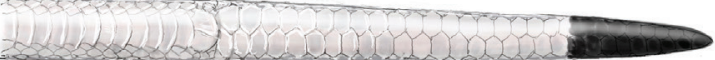

Figure I. Dorsal, lateral and ventral illustrations of previously recognized taxa, Apostolepis dimidiata (A) and $A$. barrioi (B), according to the diagnoses of Cabral et al. (2017). However, these represent merely phenotypic variations of $A$. dimidiata and, according to the descriptions of Jan (1862) and Lema (1978), both holotypes of $A$. barrioi and $A$. dimidiata present the bottom coloration.

presents a plate that encompasses all known ventral pattern variation for $A$. dimidiata, with a gradual change from immaculate yellow to black ventral patterns.

Considering that Apostolepis barrioi and A. dimidiata share the same morphological features and variation (Fig. 1), present virtually identical geographic distribution, and both descriptions are based on specimens that have the same ventral coloration, which was erroneously cited as "diagnostic" at the time, we argue that $A$. barrioi Lema, 1978 should be relegated as a junior synonym of $A$. dimidiata (Jan, 1862). The work of Cabral et al. (2017) seems to follow a recent, genus-wide trend, in which several species have been described based on poorly supported diagnoses (e.g. Apostolepis mariae Borges-Nojosa et al. 2017 (2016); A. roncadori Lema, 2016; A. thalesdelemai Borges-Nojosa et al. 2016 (2017); A. underwoodi Lema \& Campbell, 2017). None of these studies presented descriptions of osteology or hemipenes, nor do they include molecular support for their proposed species, relying exclusively upon highly variable morphological characters such as coloration and body shape. It is also noteworthy that, $A$. barrioi was described, synonymized, and then revalidated by the same author over a timespan of almost 40 years.

\section{Final remarks}

Unfortunately, several interest conflicts among researchers in the past decades have caused strong instability in Apostolepis, as well as most Elapomorphini taxa (Fig. 2). Here we present an overview of the currently recognized species of Apostolepis as well as their known specimens and diagnoses. It is noteworthy that poor diagnoses and small type series are usually associated with previous synonymizations (Table 1). We urge our fellow authors 
Table I. Valid species of Apostolepis Cope, 1862 up to date. Characters present on diagnosis: Coloration (CO), Meristic (ME), Morphometric (MO), Subjective character states related to external morphology (SU), Molecular Data (MD), Internal Morphology (IM). References: ${ }^{1}$ França et al. 2018; ${ }^{2}$ Lema 2002b; ${ }^{3}$ Peters 1869; ${ }^{4}$ Ferrarezzi et al. 2005; ${ }^{5}$ Rodrigues 1993 ; ${ }^{6}$ Reinhardt $1861 ;{ }^{7}$ Peracca $1904 ;{ }^{8}$ Harvey et al. 2001; ' $\mathrm{G}$ omes 1915; ${ }^{10}$ Lema 2003; ${ }^{11}$ Lema 2002; ${ }^{12}$ Jan $1862 ;{ }^{13}$ Schlegel $1837 ;{ }^{14}$ Duméril et al. 1854; ${ }^{15}$ Prado 1942; ${ }^{16}$ Koslowsky 1898; ${ }^{17}$ Santos et al. $2018 ;{ }^{18}$ Cope $1887 ;{ }^{19}$ Gomes in Amaral $1921 ;{ }^{20}$ BorgesNojosa et al. 2017; ${ }^{21}$ Harvey 1999; ${ }^{22}$ Lema and Renner 2004; ${ }^{23}$ Amaral 1935; ${ }^{24}$ Peters $1869 ;{ }^{25}$ Boulenger 1896; ${ }^{26}$ Amaral 1922; ${ }^{27}$ Boulenger $1903 ;{ }^{28}$ Giraudo and Scrocchi $1998 ;{ }^{29}$ Lema and Renner 2006; ${ }^{30}$ Lema 2004a; ${ }^{31}$ Ruthven 1927; ${ }^{32}$ Lema 2004b; ${ }^{33}$ Lema 2016; ${ }^{34}$ Lema and Campbell 2017.

\begin{tabular}{|c|c|c|c|c|c|c|c|c|c|}
\hline Taxon & $\begin{array}{c}\text { Year of } \\
\text { description }\end{array}$ & $\begin{array}{l}\text { Individuals in } \\
\text { type series }\end{array}$ & $\begin{array}{c}\text { Previously } \\
\text { synonymized? }\end{array}$ & $\mathrm{CO}$ & ME & MO & SU & MD & IM \\
\hline Apostolepis adhara ${ }^{1}$ & 2018 & 2 & No & + & + & + & - & - & + \\
\hline Apostolepis albicollaris ${ }^{2}$ & 2002 & 28 & No & + & + & + & + & - & - \\
\hline Apostolepis ambinigra ${ }^{3}$ & 1869 & 1 & No & + & + & + & - & - & - \\
\hline Apostolepis ammodites ${ }^{4}$ & 2005 & 25 & No & + & + & + & + & - & + \\
\hline Apostolepis arenaria & 1993 & 4 & No & + & + & + & - & - & - \\
\hline Apostolepis assimilisi ${ }^{6}$ & 1861 & $1(\geq)$ & No & + & + & - & - & - & - \\
\hline Apostolepis borelli & 1904 & 1 & Yes & + & + & + & - & - & - \\
\hline Apostolepis breviceps $^{8}$ & 2001 & 4 & No & + & + & + & + & - & - \\
\hline Apostolepis cearensis ${ }^{9}$ & 1915 & 7 & No & + & + & + & - & - & - \\
\hline Apostolepis cerradoensis ${ }^{10}$ & 2003 & 1 & No & + & + & + & + & - & - \\
\hline Apostolepis christineae ${ }^{11}$ & 2002 & 1 & No & + & + & + & + & - & - \\
\hline Apostolepis dimidiata ${ }^{12}$ & 1862 & 1 & Yes & + & + & - & + & - & - \\
\hline Apostolepis dorbignyi ${ }^{13}$ & 1837 & 1 & No & + & + & + & - & - & - \\
\hline Apostolepis flavotorquata ${ }^{14}$ & 1854 & 1 & Yes & + & + & + & - & - & - \\
\hline Apostolepis gaboi & 1993 & 1 & No & + & + & + & - & - & - \\
\hline Apostolepis goiasensis ${ }^{15}$ & 1942 & 1 & Yes & + & + & + & + & - & - \\
\hline Apostolepis intermedia ${ }^{16}$ & 1898 & 1 & No & + & + & + & - & - & - \\
\hline Apostolepis kikoi ${ }^{17}$ & 2018 & 5 & No & + & + & + & - & - & + \\
\hline Apostolepis lineata ${ }^{18}$ & 1887 & 1 & Yes & + & - & - & - & - & - \\
\hline Apostolepis longicaudata ${ }^{19}$ & 1921 & 1 & No & + & + & + & + & - & - \\
\hline Apostolepis mariae $e^{20}$ & 2017 & 22 & No & + & + & + & + & - & - \\
\hline Apostolepis multicincta ${ }^{21}$ & 1999 & 3 & No & + & + & + & - & - & - \\
\hline Apostolepis nelsonjorge $i^{22}$ & 2004 & 7 & No & + & + & + & + & - & - \\
\hline Apostolepis niceforoi ${ }^{23}$ & 1935 & 1 & No & + & + & + & + & - & - \\
\hline Apostolepis nigrolineata ${ }^{24}$ & 1869 & 1 & Yes & + & + & + & - & - & - \\
\hline Apostolepis nigroterminata ${ }^{25}$ & 1896 & 1 & No & + & + & + & - & - & - \\
\hline Apostolepis phillipsi $i^{21}$ & 1999 & 1 & No & + & + & + & - & - & - \\
\hline Apostolepis polylepis ${ }^{26}$ & 1922 & 4 & No & + & + & + & + & - & - \\
\hline Apostolepis pymi ${ }^{27}$ & 1903 & 1 & Yes & + & + & + & - & - & - \\
\hline Apostolepis quinquelineata ${ }^{25}$ & 1896 & 1 & Yes & + & + & + & - & - & - \\
\hline Apostolepis quirogai ${ }^{28}$ & 1998 & 2 & No & + & + & + & - & - & - \\
\hline Apostolepis serrana $a^{29}$ & 2006 & 1 & No & + & + & + & + & - & - \\
\hline Apostolepis striata ${ }^{30}$ & $2004 a$ & 1 & No & + & + & + & + & - & - \\
\hline Apostolepis tenuis $^{31}$ & 1927 & 1 & Yes & + & + & + & - & - & - \\
\hline Apostolepis tertulianobeui ${ }^{32}$ & $2004 b$ & 1 & No & + & + & + & + & - & - \\
\hline Apostolepis thalesdelemai ${ }^{20}$ & 2017 & 15 & No & + & + & + & + & - & - \\
\hline Apostolepis roncadori ${ }^{33}$ & 2016 & 1 & No & + & + & + & + & - & - \\
\hline Apostolepis underwoodi $i^{34}$ & 2017 & 3 & No & + & + & + & + & - & - \\
\hline Apostolepis vittata ${ }^{18}$ & 1887 & 1 & Yes & + & - & - & - & - & - \\
\hline
\end{tabular}




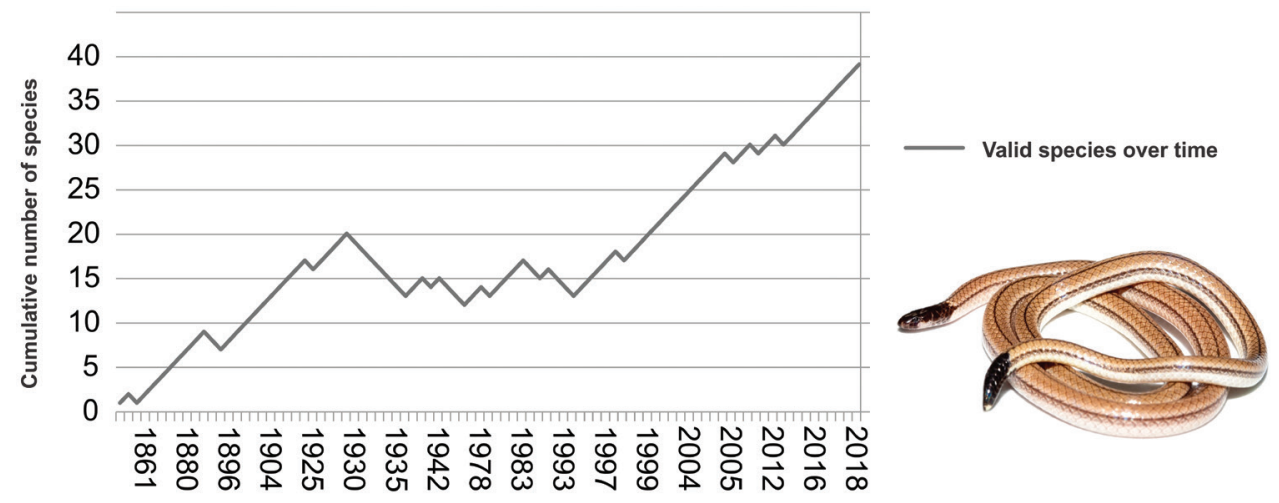

Timespan in years

Figure 2. The impact of the taxonomic history of Apostolepis Cope, 1862 species. The line refers to the cumulative number of species considered as valid during the time span, suffering either reductions from synonymies or additions from descriptions and revalidations. Inset picture: Apostolepis sp. from Serra do Cachimbo, Pará, Brazil.

not to commit taxonomic malpractice and to carefully generate, rethink and analyze their data, providing compelling evidence for their claims. The careless proliferation and splitting of taxa may present deleterious consequences not only to the field of taxonomy but also to directing conservation efforts. An integrative revision, preferably incorporating aspects of external and internal morphology, along with molecular data, is largely warranted in order to mitigate and reevaluate the taxonomy of Apostolepis as a whole.

\section{Acknowledgments}

We thank Peter Uetz (VCU) and Henrique Caldeira Costa (UFV), who kindly provided us with some rare literature. We are also deeply indebted to Tarcísio Lyra dos Santos Abreu (UnB) and Russell Gray (UF-IFAS) for helping troubleshoot our analyses, and to Editor Robert Jadin (UW-Eau Claire) and Francisco Franco (IBSP), who provided constructive criticism and comments on our manuscript. Omar M. Entiauspe-Neto and D. Loebmann thank CNPq for a PIBIC grant (136628/2016-8); D. Loebmann is a Research Fellow of CNPq (Proc. \#310651/2017-4).

\section{References}

Amaral A (1921) Duas novas espécies de Colubrideos opysthoglyphos brasileiros (Philodryas oligolepis e Apostolepis longicaudata). Annaes Paulistas de Medicina \& Cirurgia 9: 3-6.

Amaral A (1922) Contribuição para o conhecimento dos ofídios do Brazil. Parte I Quatro novas espécies de serpentes brasileiras. Memórias do Instituto Butantan 1[1921]: 1-37. 
Amaral A (1935) Estudos sobre ophídios neotrópicos XXXIII. Novas espécies de ophídios do Colômbia. Memórias do Instituto Butantan 9: 219-223.

Borges-Nojosa DM, Lima DC, Bezerra CH, Harris JH (2017) Two new species of Apostolepis Cope, 1862 (Serpentes: Elapomorphini) from brejos de altitude in Northeastern Brazil. Revista Nordestina de Zoologia 10[2016]: 74-94.

Boulenger GA (1896) Catalogue of snakes in the British Museum, Vol. 3. Taylor and Francis, London.

Boulenger GA (1903) Descriptions of new snakes in the collection of the British Museum. Annals \& Magazine Natural History 12: 350-354. https://doi.org/10.1080/00222930308678866 Cabral H, Lema T, Renner MF (2017) Revalidation of Apostolepis barrioi (Serpentes: Dipsadidae). Phyllomedusa 16: 243-254. https://doi.org/10.11606/issn.2316-9079.v16i2p243-254

Cei JM (1993) Reptiles del noroeste, nordeste y este de la Argentina. Herpetofauna de las Selvas Subtropicales, Puna y Pampas. Museo Regionale di Scienzi Naturali, Torino, Monografia XIV. Cope ED (1862) "Permission was granted to Mr. E. D. Cope to State to the Academy a few observations ... [Notes on Elapomorphus and Coniophanes]". Proceedings of the Academy of Natural Sciences of Philadelphia 13[1861]: 522-524.

Cope ED (1887) Synopsis of the Batrachia and Reptilia obtained by H. H. Smith in the Province of Mato Grosso, Brazil. Proceedings of the American Philosophical Society 24: 44-60.

Duméril AMC, Bibron G, Duméril AHA (1854) Erpétologie géneralé ou histoire naturelle complète des reptiles. Tome septième. Deuxième partie, comprenant l'histoire des serpentes venimeux. Librairie Encyclopédique de Roret, Paris, 781-1536.

Ferrarezzi H (1993) Sistemática Filogenética de Elapomorphus, Phalotris e Apostolepis (Serpentes: Colubridae: Xenodontinae). MSc. dissertation, Departamento de Zoologia, Instituto de Biociências, Universidade de São Paulo, São Paulo, 277 pp.

Ferrarezzi H, Barbo FE, Albuquerque CE (2005) Phylogenetic relationship of a new species of Apostolepis from Brazilian Cerrado with notes on the assimilis group (Serpentes: Colubridae: Xenodontinae: Elapomorphini). Papéis Avulsos de Zoologia 45: 215-229. https://doi. org/10.1590/S0031-10492005001600001

França DPF, Barbo FE, Silva-Júnior NJ, Silva HLR, Zaher H (2018) A new species of Apostolepis (Serpentes, Dipsadidae, Elapomorphini) from the Cerrado of Central Brazil. Zootaxa 4521: 539-552. https://doi.org/10.11646/zootaxa.4521.4.3

Giraudo A, Scrocchi GJ (1998) A new species of Apostolepis (Serpentes: Colubridae) and comments on the genus in Argentina. Herpetologica 54: 470-476.

Gomes JF (1915) Contribuição para o conhecimento dos ophidios do Brasil. 1. Descrição de quatro espécies novas e um novo Gênero de opisthóglyphos. 2. Ophidios do Museu Rocha (Ceará). Annais Paulistas Medicina \& Cirurgia 4: 121-129.

Harvey MB (1999) Revision of Bolivian Apostolepis (Squamata: Colubridae). Copeia 1999: 388-409. https://doi.org/10.2307/1447485

Harvey MB, Lucindo Gonzales A, Scrocchi GJ (2001) New species of Apostolepis (Squamata: Colubridae) from Gran Chaco in Southern Bolivia. Copeia 2001: 501-507. http://doi. org/10.1643/0045-8511(2001)001[0501:NSOASC]2.0.CO;2

Jan G (1862) Enumerazione sistematico delle specie d'ofidi del gruppo Calamaridae. Archivio per la Zoologia l'Anatomia e la Fisiologia 2: 1-76. 
Koslowsky J (1898) Ofidios de Matto-Grosso (Brazil). Revista del Museu de La Plata 8: 25-34. Lema T (1978) Novas espécies de opistoglifodontes do gênero Apostolepis Cope, 1861 do Paraguai (Ophidia: Colubridae: Colubrinae). Comunicaçóes do Museu de Ciências PUCRS 18/19: 27-49.

Lema T (1986) Apostolepis dimidiata (Jan, 1862), nova combinação, e a validade de A. erythronota (Peters, 1880) e A. ventrimaculata Lema, 1978. (Serpentes: Colubridae: Elapomorphinae). Memórias do Instituto Butantan 47-48: 71-80.

Lema T (1993) Polimorfismo em Apostolepis dimidiata (Jan, 1862), com a invalidação de Apostolepis villaricae Lema, 1978 e Apostolepis barrioi Lema, 1978 (Serpentes: Colubridae: Xenodontinae: Elapomorphini). Acta Biologica Leopoldensia 15: 35-52.

Lema T (2002a) Nova espécie de Apostolepis do grupo lineata do sudoeste do Brasil (Serpentes, Elapomorphinae). Facena 18: 41-52.

Lema T (2002b) Nova especie de Apostolepis Cope do grupo dimidiata do Cerrado do Brasil (Serpentes: Elapomorphinae). Comunicaçóes do Museu de Ciências e Tecnologia da PUCRS Serie Zoologia 15(2): 227-238.

Lema T (2003) Descrição de nova espécie de Apostolepis Cope do Cerrado do Brasil, pertencente ao grupo dimidiata (Serpentes, Elapomorphinae). Acta Biologica Leopoldensia 25: $123-131$.

Lema T (2004b) Description of a new species of Apostolepis Cope, 1861 (Serpentes, Elapomorphinae) from Brazilian Cerrado. Acta Biologica Leopoldensia 26: 155-160.

Lema T (2004a) Nova espécie de Apostolepis COPE do estado de Rondônia, Brasil (Serpentes, Elapomorphinae). Comunicaçóes do Museu Ciência Tecnologia (PUCRS), Porto Alegre 17: 81-89.

Lema T, Renner MF (2004) New species of Apostolepis from Alto Tocantins, Brazil, with comments on the striped pattern species (Serpentes, Elapomorphinae). Biociências 12: 139-145.

Lema T, Renner MF (2006) A new species of Apostolepis with striped pattern from Mato Grosso, Brasil (Serpentes, Elapomorphine). Ciência Em Movimento 8: 13-18.

Lema T (2016) Description of new species of Apostolepis (Serpentes: Dipsadidae: Xenodontinae: Elapomorphini) from Serra do Roncador, Central Brazil. Caderno de Pesquisa, série Biologia 28: 1-12.

Lema T, Campbell P (2017) New Species of Apostolepis (Serpentes, Dipsadinae, Elapomorphini) from Bolivia, from the Apostolepis borellii group. Research \& Reviews: Journal of Zoological Sciences 5(1): 19-28.

Nogueira C, Barbo FE, Ferrarezzi H (2012) Redescription of Apostolepis albicollaris Lema, 2002, with a Key for the Species Groups of the Genus Apostolepis (Serpentes: Dipsadidae: Elapomorphini). South America Journal of Herpetology 7: 213-225. https://doi. org/10.2994/057.007.0303

Peracca MG (1904) Viaggio del Dr. A. Borelli nel Matto Grosso brasiliano e nel Paraguay, 1899. IX. Rettili ed amfibii. Bollettino dei Musei di Zoologia ed Anatomia comparata della R. Università di Torino 19: 1-15.

Peters WCH (1869) Über neue Gattungen und neue oder weniger bekannte Arten von Amphibien (Eremias, Dicrodon, Euprepes, Lygosoma, Typhlops, Eryx, Rhynconix, Elapomorphus, Achalinus, Coronella, Dromicus, Xenopholis, Anoplodipsas, Spilotes, Tropidonotus). Monatsberichte der Königlichen Preussiche Akademie des Wissenschaften zu Berlin, 432-447. 
Peters WCH (1880) Eine Mittheilung über neue oder weniger bekannte Amphibien des Berliner Zoologischen Museums (Leposoma dispar, Monopeltis (Phractogonus jugularis, Typhlops depressus, Letpocalamus drilineatus, Xenodon punctatus, Elapomorphus erythronotus, Hylomantis). Monatsberichte der Königlichen Preussiche Akademie des Wissenschaften zu Berlin, 217-224.

Prado A (1942) Notas ofiológicas. 14. Comentários acerca de algumas serpentes opistóglifas do gênero Apostolepis, com a descrição de uma nova espécie. Memórias do Instituto Butantan 16: 7-12.

Reinhardt (1861) Herpetologiske Middelelser. II. Beskrivelser af nogle nye til Calamariernes Familie henhörende Slänger. Videnskabelige meddelelser fra den Naturhistoriske forening i Kjöbenhavn: 229-250.

Rodrigues MT (1993) Herpetofauna das dunas interiores do Rio São Francisco: Bahia: Brasil. V. Duas novas espécies de Apostolepis (Ophidia, Colubridae). Memórias Instituto Butantan 54: 53-59.

Ruthven AG (1927) Description of an apparently new species of Apostolepis from Bolivia. Occasional Papers of the Museum of Zoology, University of Michigan 188: 1-2.

Santos FM dos, Entiauspe-Neto OM, Araújo JS, de Souza MB, de Lema T, Strussmann C, de Albuquerque NR (2018) A new species of burrowing snake (Serpentes: Dipsadidae: Apostolepis) from the state of Mato Grosso, Central-West region of Brazil. Zoologia 35: 1-10. https://doi.org/10.3897/zoologia.35.e26742

Schlegel H (1837) Essai sur la physionomie des serpens.Partie Descriptive. J. Kips, J. HZ. et W.P. van Stockum, La Haye, 606 pp.

Vanzolini PE (1986) Addenda and corrigenda to the catalogue of Neotropical Squamata. Smithsonian Herpetological Information Service 70: 1-26. https://doi.org/10.5479/ si.23317515.70.1

Werner F (1897) Ueber einige neue oder seltene Reptilien und Frösche der Zoologischen Sanmlung des Staates in München. Sitzungsberichte der Bayerischen Akademie der Wissenschaften zu München, Mathematisch-Physikalische Klasse 27: 203-220. 